\title{
Evaluation of the Radiation Pneumonia Development Risk in Lung Cancer Cases
}

\author{
Sercan Yilmaz ${ }^{1}$, Yasemin Guzle Adas ${ }^{2 *}$, Ayse Hicsonmez ${ }^{3}$, Meltem Nalca Andrieu ${ }^{3}$, \\ Serap Akyurek ${ }^{3}$, Saban Cakir Gokce ${ }^{3}$
}

\begin{abstract}
Background: Concurrent chemo-radiotherapy is the recommended standard treatment modality for patients with locally advanced lung cancer. The purpose of three-dimensional conformal radiotherapy (3DCRT) is to minimize normal tissue damage while a high dose can be delivered to the tumor. The most common dose limiting side effect of thoracic RT is radiation pneumonia (RP). In this study we evaluated the relationship between dose-volume histogram parameters and radiation pneumonitis. This study targeted prediction of the possible development of RP and evaluation of the relationship between dose-volume histogram (DVH) parameters and RP in patients undergoing 3DCRT. Materials and Methods: DVHs of 41 lung cancer patients treated with 3DCRT were evaluated with respect to the development of grade $\geq 2 \mathrm{RP}$ by excluding gross tumor volume (GTV) and planned target volume (PTV) from total (TL) and ipsilateral (IPSI) lung volume. Results: Were admitted statistically significant for $\mathbf{p}<\mathbf{0 . 0 5}$. Conclusions: The cut-off values for V5, V13, V20, V30, V45 and the mean dose of TL-GTV; and V13, V20,V30 and the mean dose of TL-PTV were statistically significant for the development of Grade $\geq 2$ RP. No statistically significant results related to the development of Grade $\geq 2$ RP were observed for the ipsilateral lung and the evaluation of PTV volume. A controlled and careful evaluation of the dose-volume histograms is important to assess Grade $\geq 2 \mathrm{RP}$ development of the lung cancer patients treated with concurrent chemo-radiotherapy. In the light of the obtained data it can be said that RPdevelopment may be avoided by the proper analysis of the dose volume histograms and the application of optimal treatment plans.
\end{abstract}

Keywords: Lung cancer - radiotherapy - radiation pneumonitis - prevention

Asian Pac J Cancer Prev, 15 (17), 7371-7375

\section{Introduction}

Lung cancer is one of the most commonest and most lethal type of cancer worldwide. For the treatment of lung cancer, surgery is used in the early stages and chemoradiotherapy (CRT) is used in the locally advanced stages. RT has an important role in the treatment of lung cancer. (Liu et al., 2013; Rendan et al., 2013; Malik et al 2014).

Radiation Therapy has extensive application in current pulmonary neoplasms treatment. Therefore, potential side effects of RT should be observed during and after the treatment. Among these side effects, the most important and dose-limiting one is the radiation pneumonia (RP) (Wang et al., 2012).

With the use of 3DCRT, the correlation between RP and several dosimetric parameters was examined. In previous studies, it was predicted that the use of 3DCRT resulted in a risk of 13-37\% for RP. Based on the literature, despite the presence of numerous studies that evaluated radiation pneumonia, there is not an ideal dose-volume value for the prediction of RP (Mehta et al., 2005). The examined parameters include volume 20 (V20), volume 30 (V30) and mean lung dose (MLD) that were measured by considering both lungs as one single organ. In the studies performed, the risk for RP development was tried to be determined using V20, V30 and MLD among DVH parameters in 3DCRT (Lawrence et al., 1998, Hernando et al., 2001). As both lungs were considered as a single functional unit, potential imbalance of the dose distribution between ipsilateral and contrlateral lung was not taken into consideration. In the previous studies, there was no clear information about the extraction of PTV or GTV from total lung volume.Due to the PTV and GTV volumes, normal lung volume and related DVH parameters may be variant.

This study aimed to reach an ideal dose-volume parameter that is effective on the development of RP.

\section{Materials and Methods}

This study is conducted retrospectively using the

${ }^{1}$ Department of Radiation Oncology, Diyarbakır State Hospital, Diyarbakır, ${ }^{2}$ Department of Radiation Oncology, Manisa State Hospital, Manisa, ${ }^{3}$ Department of Radiation Oncology, Medical School, Ankara University, Ankara, Turkey *For correspondence: yaseminadas@gmail.com 
information recorded in the treatment planning systems (TPS) for 41 patients with lung cancer who were treated with chemoradiotherapy in Ankara University Medical School Radiation Oncology Department between February 2009 and July 2011. Patient characteristics are summarised in Table 1.

In the contouring of the target volume ICRU 50/62 was used. GTV was contoured to include primary tumor and lymph nodes involved (GTV LN). As done with standard therapeutic approach, both lungs were contoured as a single organ and GTV was excluded from this total lung volume. For CTV, a margin of $1-1,5 \mathrm{~cm}$ to GTV and for PTV a margin of $0,5-1 \mathrm{~cm}$ to CTV was established on the planning computer in all directions. CTV and PTV margins were modified according to patients' clinics and critical organ tolerance doses. 3D-CRT was conducted on using the planning program of Precise Plan Release 2.16

Appropriate dose-volume histograms were obtained in a manner not to exceed critical organ tolerance doses (M. Spinalis <46 Gy; esophagus V55 <50\%, heart V40 $<50 \%$ ). All patients were underwent therapy on this plan using Lineer accelerator (Elekta Synergy Platform) with photon energies of $6 \mathrm{Mv}-18 \mathrm{Mv}$.

In the planning system, GTV and PTV volumes were extracted from TL and IPSI lung (the lung in which primary tumor is localized and more than $50 \%$ is involved in the treatment volume is considered as ipsilateral lung) and DVHs were obtained. Based on these DVHs, TL-GTV, TL-PTV, IPSI-GTV, IPSI-PTV dose and volumes, V5, V13, V20, V20, V30, V45 percentages and MLD were obtained for each patient. The effect of these results on radiation pnemunia development was eveluated

\section{Statistical method}

The data were analyzed using SPSS for Windows 11.5. In order to determine whether there is a difference of demographics and disease characteristics between the patients with and without RP, Chi-square and Fisher's Exact Test were used. For the pulmonary measurements (PTV, GTV), median of the patients' results were taken and cut-off values were determined. Fisher's Exact Test was used in order to determine whether these values were different between the patients with and without RP. In the evaluations performed among all subjects, $\mathrm{p}<0.05$ was considered as statistically significant.

\section{Results}

Mean follow-up was 11.7 months. Mean overall survival was 15.3 months, disease-free survival was $24.4 \%$ and overall survival was $39.1 \%$. While $87.8 \%$ of the patients (36 patients) did not have RP, 5 patients $(12.2 \%$ ) had RP. From the initiation of RT, mean RP occurrence time was found to be 6.8 months.

No statistically significant difference was found for RP development between the groups with and without RP in terms of TL-GTV mean dose (MLD), TL-GTV volume, TL-GTV V20, and TL-GTV V30. The incidence of RP was significantly lower in the patients with a cut-off value of TL-GTV mean dose $<2012$ cGy $v s>2012$ cGy ( $\mathrm{p}=0.04$, Table 2$)$; in the patients with a cut-off value of
TL-GTV V20 <37\% vs V20 >37\% ( $\mathrm{p}=0.04$, Table 2); in the patients with a cut-off value of TL-GTV V30 <32\% vs $\mathrm{V} 30 \geq 32 \%$ ( $\mathrm{P}=0.04$, Table 2$)$; in the patients with a cut-off value of TL-GTV V5 below 56\% compared to those with the corresponding value above $56 \%(\mathrm{p}=0.04$, Table 2); in the patients with a cut-off value of TL-GTV $\mathrm{V} 13<48 \%$ vs V13 $>48 \%$ ( $\mathrm{p}=0.04$, Table 2); in the patients with a cut-off value of TL-GTV V45 <24\% vs V45 $\geq 24 \%$ ( $\mathrm{p}=0.04$, Table 2 ). No statistically significant difference of RP development was found between two groups when cut-off value for TL-GTV was considered to be $3462 \mathrm{cc}$ and cut-off value for TL-PTV volume was considered to be $3259 \mathrm{cc}$.

When the patients were evaluated based on the results obtained by extracting PTV from TL volume, there was no statistically significant difference for mean TL-PTV dose, TL-PTV volume, TL-PTV V20 and TL-PTV V30 between the groups with and without RP.

The incidence of RP was significantly lower in the patients with a cut-off value of TL-PTV mean dose $<1849$ cGy $v s \geq 1849$ cGy ( $\mathrm{p}=0.04$, Table 2$)$.

When V20 $<31 \%$ and V30<25\%, which is recommended in standard therapies, were evaluated in our study, a statistical difference was found in TL-PTV volumes. The incidence of RP was significantly lower in the patients with TL-PTV V20 $<31 \%$ and TL-PTV V30 $<25 \%$ compared to higher values ( $\mathrm{p}=0.04$, Table 2$)$. In addition, cut-off value of TL-PTV V30 was found to be $25 \%$, which is consistent with the literature.

The incidence of RP was significantly lower in the patients with a cut-off value of TL-PTV V13 $<43 \%$ vs V13 $\geq 43 \%$ ( $\mathrm{p}=0.04$, Table 2$)$.

No difference of RP incidence was found in the patients with a cut-off value of TL-PTV V $5<53 \%$ vs V 5 $\geq 53 \%$ and in the patients with a cut-off value of TL-PTV $\mathrm{V} 45<17 \%$ vs $\mathrm{V} 45>17 \%$.

When the patients were evaluated according to the values obtained from ipsilateral lung, no statistically significant difference was recorded for mean IPSI-PTV

\section{Table 1. Patient Characteristics}

\begin{tabular}{|c|c|c|c|}
\hline & & $\begin{array}{c}\text { Number of } \\
\text { patients }\end{array}$ & $\begin{array}{c}\text { Percentage of } \\
\text { patients }\end{array}$ \\
\hline \multirow[t]{2}{*}{ Age } & $\leq 57$ & 21 & 51.2 \\
\hline & $>57$ & 20 & 48.8 \\
\hline \multicolumn{4}{|c|}{ Performance score } \\
\hline & $0-1$ & 34 & 82.9 \\
\hline & $\geq 2$ & 7 & 17.1 \\
\hline \multicolumn{4}{|c|}{ Pathology } \\
\hline & Non small cell & 26 & 63.4 \\
\hline & Small cell & 15 & 36.6 \\
\hline \multirow[t]{3}{*}{ Stage } & I (Small cell ca) & 15 & 36.6 \\
\hline & II (Non-small cell ca) & 1 & 2.4 \\
\hline & III (Non-small cell ca) & 25 & 61 \\
\hline \multicolumn{4}{|c|}{ Radiation Dose } \\
\hline & $\leq 6000$ cGy & 29 & 70.7 \\
\hline & $>6000$ cGy & 12 & 29.3 \\
\hline \multicolumn{4}{|c|}{ Chemotherapy } \\
\hline & Concurent & 15 & 36.6 \\
\hline & Adjuvant & 1 & 2.4 \\
\hline & Neoadjuvant+concurent & 25 & 61 \\
\hline
\end{tabular}


Table 2. Results

\begin{tabular}{|c|c|c|c|}
\hline & $\mathrm{RP}(-)$ & $\mathrm{RP}(+)$ & p-value \\
\hline \multicolumn{4}{|c|}{ TL-GTV mean dose 2012 cGy } \\
\hline$<2012$ & $100 \%$ & $0.00 \%$ & 0.04 \\
\hline$\geq 2012$ & $75 \%$ & $25 \%$ & \\
\hline \multicolumn{4}{|c|}{ TL-GTV V20 \%37 } \\
\hline$<37$ & $100 \%$ & $0.00 \%$ & 0.04 \\
\hline$\geq 37$ & $76.50 \%$ & $23.50 \%$ & \\
\hline \multicolumn{4}{|c|}{ TL-GTV V30 32\% } \\
\hline$<32$ & $100 \%$ & $0.00 \%$ & 0.04 \\
\hline$\geq 32$ & $78.90 \%$ & $21.10 \%$ & \\
\hline \multicolumn{4}{|c|}{ TL-GTV V5 56\% } \\
\hline$<56$ & $100 \%$ & $0 \%$ & 0.04 \\
\hline$\geq 56$ & $76.50 \%$ & $23.50 \%$ & \\
\hline \multicolumn{4}{|c|}{ TL-GTV V13 48\% } \\
\hline$<48$ & $100 \%$ & $0 \%$ & 0.04 \\
\hline$\geq 48$ & $76.50 \%$ & $23.50 \%$ & \\
\hline \multicolumn{4}{|c|}{ TL-GTV V45 24\% } \\
\hline$<24$ & $100 \%$ & $0 \%$ & 0.04 \\
\hline$\geq 24$ & $76.50 \%$ & $23.50 \%$ & \\
\hline \multicolumn{4}{|c|}{ TL-PTV mean dose 1849 cGy } \\
\hline$<1849$ & $100 \%$ & $0 \%$ & 0.04 \\
\hline$\geq 1849$ & $76.50 \%$ & $23.50 \%$ & \\
\hline \multicolumn{4}{|c|}{ TL-PTV V20 31\% } \\
\hline$<31$ & $100 \%$ & $0 \%$ & 0.04 \\
\hline$\geq 31$ & $78.90 \%$ & $21.10 \%$ & \\
\hline \multicolumn{4}{|c|}{ TL-PTV V30 25\% } \\
\hline$<25$ & $100 \%$ & $0 \%$ & 0.04 \\
\hline$\geq 25$ & $78.90 \%$ & $21.10 \%$ & \\
\hline \multicolumn{4}{|c|}{ TL-PTV V13 43\% } \\
\hline$<43$ & $100 \%$ & $0 \%$ & 0.04 \\
\hline$\geq 43$ & $76.50 \%$ & $23.50 \%$ & \\
\hline
\end{tabular}

dose, IPSI-PTV volume, IPSI-PTV V20, IPSI-PTV V30, IPSI-GTV dose, IPSI-GTV volume, IPSI-GTV V20 and IPSI-GTV V30 in terms of RP development. IPSI-GTV could not be evaluated in 8 patients because 2 underwent pneumonectomy and 6 underwent lobectomy.

When the patients were evaluated in terms of PTV volume, no statistically significant difference was found between the groups with and without RP.

\section{Discussion}

Lung cancer is the most commonly seen cancer type and the leading cause of cancer-related death cases. An important part of the primary therapy in locally advanced disease includes radiotherapy. In thoracic RT, most commonly observed dose-limiting toxicity is radiation-related pulmonary injury. The introduction of three-dimensional planning systems allowed the clinician to examine the detailed dose distributions by obtaining dose-volume histograms. Many investigators developed various models in order to reveal the correlation between dose-volume parameters and normal tissue injury. Most appropriate RP-related dosimetric factors that were published included MLD, V20 and V30 (Fay et al., 2005).

In order to determine appropriate dose-volume value for RP, some investigators considered the lungs as a single organ, whereas others evaluated the lung as both a single organ and two separate organs. While some studies excluded GTV from the lung volume, others excluded PTV from the lung volume (Rancati et al., 2003;
Schallenkamp et al., 2007; Ramella et al 2010).

In this study, we excluded GTV and PTV from both total lung and ipsilateral lung in order to establish a correlation with RP.

In this study, based on our standard planning (TLGTV), we detected grade $\geq 2 \mathrm{RP}$ in 5 of 41 patients (12.2\%). We found MLD as $1925 \mathrm{cGy}$ and we did not find a significant correlation between the development of grade $\geq 2$ RP and MLD ( $\mathrm{p}=0.07$ ). The fact that MLD was above the cut-off value, $2012 \mathrm{cGy}$, was found to be significant in terms of RP development $(\mathrm{p}=0.04)$. Rancati et al., excluded GTV from lung volume by considering the lung as a single organ and evaluated MLD in terms of RP. In 14 of 84 patients $(16.7 \%)$, the development of grade $\geq 2$ RP was detected. Similar to our results, no correlation was detected between MLD and RP development ( $p>0,4)$ (Rancati et al., 2003).

In a study performed by Feng-Ming et al., on 109 patients with NSCLC, GTV was exctracted from total lung volume and 17 patients $(14,6 \%)$ were found to have grade 2 and grade 3 RP. In the univariate analysis, MLD was found to be significant for RP development; this rate was found to be $3.9 \%$ when MLD was <14 Gy and 32.6\% when MLD was $>14$ Gy ( $\mathrm{p}<0.002)$ (Kong et al., 2006).

In this study, although the cut-off value of MLD for RP development was higher compared to other studies, as reported in the study performed by Hernando et al. (2001) increased MLD led to an increase in the risk for RP development and this rate was found to be $27 \%$ when the dose ranged between 21-30 Gy. In the study of Hernando GTV was extracted from total lung volume (Hernando et al., 2001).

In this study, we determined V20, V30 values to establish the risk for RP. Although in the literature, V20 is a widely used parameter for the determination of the risk for RP, there are conflicts about its correlation with RP. V20 rate was $36 \%$ in the group without RP and $41.5 \%$ in the group with RP. V30 rate was $30.1 \%$ in the group without RP and $34.7 \%$ in the group with RP and V20 and $\mathrm{V} 30$ rates were not statistically different between two groups ( $\mathrm{p}=0.25, \mathrm{p}=0.18)$. V20 cut-off value was found to be $37 \%$, which is a statistically significant value for RP development $(\mathrm{p}=0.04)$. In a study performed by Tsujino et al., 71 patients with lung cancer were evaluated and GTV was exctracted from total lung volume. At the end of a mean follow-up of 7.5 months, in 20 patients $(28 \%)$ grade $\geq 2$ RP was observed (6-month, 27.3\%; 12-month, $31.2 \%)$. In all patients, mean V20 rate was found to be $23 \%$. Increasing V20 value was reported to be associated with RP. The incidence of RP was $8 \%$ when V $20 \leq 20 \%$; $18 \%$ when V20 ranged between $21-25 \% ; 51 \%$ when V20 ranged between $26-30 \%$ and $85 \%$ when V20 >31\% and a statistically significant difference was obtained $(p<0.0001)$ (Tsujino et al., 2003). These results were quite lower compared to our results. This may be explained by the lack of elective nodal irradiation in our study. GTV volume was smaller and therefore, normal tissue volume from which GTV extracted was greater.

Similarly, V30 values also differ. In a study performed by $\mathrm{Fu}$ et al. (2001) with 103 patients, development of symptomatic RP was examined and, at the end of 2-year 
follow-up, 16 patients (21\%) were found to have RP. For V30, there was no statistical difference in terms of RP development (Fu et al., 2001). In a study performed by Rancati et al., univariate analysis showed that V20 and V30 were significant for RP development $(\mathrm{p}<0.05)$. It was highlighted that V20 was the most powerful marker of RP development when mitomycin was used(Rancati et al., 2003). In this study, we could not have such a result because we did not evaluate chemotherapy agents.

Although DVH parameters examined for the risk of RP development generally include MLD, V20 and V30, some investigators evaluated V5, V13 and V45 during the last years (Kong et al., 2006; Wang et al., 2006). As it is difficult to determine the risk for RP using only one DVH parameter, the evaluation of the values at multiple points of the DVH curves was suggested. This led to the discussion about the correlation between the large volume exposed to low dose and small volume exposed to high dose.

In this study, the incidence of grade $\geq 2 \mathrm{RP}$ was found to be significantly lower in the patients with a cut-off value for $\mathrm{V} 5<56 \%$ vs $\geq 56 \%$ ( $\mathrm{p}=0.04)$. The incidence of grade $\geq 2$ RP was found to be significantly lower in the patients with a cut-off value for $\mathrm{V} 13<48 \%$ vs $\mathrm{V} 13 \geq 48 \%$ $(\mathrm{p}=0.04)$. Cut-off value for V45 was found to be $24 \%$. We detected less RP development when V45 < 24\% compared to $\mathrm{V} 45 \geq 24 \%(\mathrm{p}=0.04)$.

In a study performed by Wang et al (30) 223 patients who concomitantly received chemoradiotherapy were evaluated and median follow-up period of 10.5 months, grade $\geq 3 \mathrm{RP}$ was observed $22 \%$ and $32 \%$, in 6 months and 1 year respectively. In the multivariate analysis, V5 was found to be the most powerful dosimetric factor in RP development. The risk for grade $\geq 3 \mathrm{RP}$ was reported to be $3 \%$ when V $5<42 \%$ and $38 \%$ when V5 $>42 \%$ (p<0.001) (Wang et al., 2006). In a study performed by Feng-Ming et al. the risk for RP development was found to be $3 \%$ when v13<27\%; $18 \%$ when v13 ranged between $27-34 \%$ and $37 \%$ when V13 $>34 \%$. In the study of Rancati et al., univariate analysis showed that V45 was statistically significant in grade $\geq 2$ RP development (Kong et al., 2006). Our results were slightly higher than the results published in the literature, which may be based on the differences on planning and the volumes of the normal lungs that were evaluated.

In the evaluation of DVH parameters, the lung was considered to be a single functional organ, which might lead to an imbalance of dose distribution in ipsilateral and contrlateral lung. Although rarely, the localization of the tumor in one single side may result to the fact that contlateral lung does not receive any dose. Therefore, some studies evaluated DVH parameters based on a single lung (Kim et al., 2011). In this study, we considered the lung with tumor localization as ipsilateral lung, we excluded both GTV and PTV volumes from normal lung volume and we evaluated DVH parameters. Our results did not show any significant result for any DVH parameter evaluated in terms of grade $\geq 2$ RP development.

In the study performed by Kim et al., ipsilateral lung was separately evaluated and PTV was excluded from ipsilateral lung. In the univariate analysis, ipsilateral MLD, V20 and V30 values were found to be correlated with grade $\geq 2$ RP development (Kim et al., 2011). However, in this study, all patients underwent ENI and CTV was formed by giving a margin of $2-2.5 \mathrm{~cm}$ to GTV in all directions and PTV was formed by giving a margin of $0.5-1 \mathrm{~cm}$ to $\mathrm{CTV}$ in all directions. Consequently, this large volumetric contouring is different from standard contouring approach that we adopted in our clinic and it contains large margins. Therefore, as the remaining lung tissue is small, the determination of this with RP at low doses will be easier, contrary to our results.

In this study, the failure of obtaining significant data for ipsilateral lung may be explained by small number of patients, heterogeneity of the patients in terms of histological diagnosis, our CTV-PTV margins and our different chemotherapy protocols. DVH parameters of the ipsilateral lung may be new markers for the prediction of RP in the patients who receive concomitant chemoradiotherapy. However, prospective studies with larger and homogenous patient samples are warranted to include it among standard therapeutic options under ideal conditions.

In conclusion, there are many studies to determine the risk for RP using DVH parameters in the patients who received RT for the lung cancer. In the previous studies, the risk for RP development was reported to be $13 \%$ and $37 \%$. Although DVH parameters seem to be associated with the definition of this risk, it cannot reach to an adequate power to predict the development of RP.

However, the fact that DVH parameters may be evaluated using 3D planning may lower the risk for RP and allows to administer the optimal RT technique, volume and dose. Based on our results, we could reach to total dose with a RP risk rate of $12.2 \%$ by maintaining MLD at $20 \mathrm{~Gy}$ and V20 below $35 \%$ among DVH parameters, in the local RT performed by excluding ENI that we included in the therapeutic schedule by considering the lungs as a single organ after exracting GTV from total lung volume upon the pre-treatment PET in the patients with lung cancer.

At the same time, we demonstrated that the evaluation of different parameters such as V5-13-45 was also important for the prediction of RP development. These results showed our standard values used for the treatment of the patients with lung cancer.

\section{References}

Fay M, Tan A, Fisher R, et al (2005). Dose-volume histogram analysis as predictor of radiation pneumonitis in primary lung cancer patients treated with radiotherapy. International Journal of Radiation Oncology*Biology*Physics, 61, $1355-63$

Fu XL, Huang H, Bentel G, et al (2001).Predicting the risk of symptomatic radiation-induced lung injury using both the physical and biologic parameters $\mathrm{V}(30)$ and transforming growth factor beta. Int J Radiat Oncol Biol Phys, 50, 899908.

Hernando ML, Marks LB, Bentel GC, et al (2001). Radiationinduced pulmonary toxicity: a dose-volume histogram analysis in 201 patients with lung cancer. Int J Radiat Oncol Biol Phys, 51, 650-9.

Kim M, Lee J, Ha B, et al (2011). Factors predicting radiation pneumonitis in locally advanced non-small cell lung cancer. Radiat Oncol J, 29, 181-90. 
Kong FM, Hayman JA, Griffith KA, et al (2006). Final toxicity results of a radiation-dose escalation study in patients with non-small-cell lung cancer (NSCLC): Predictors for radiation pneumonitis and fibrosis, Int J Radiat Oncol Biol Physics, 65, 1075-86.

Kong FM, Hayman JA, Griffith KA, et al (2006). Final toxicity results of a radiation-dose escalation study in patients with non-small-cell lung cancer (NSCLC): Predictors for radiation pneumonitis and fibrosis Int J Radiat Oncol Biol Physics, 65, 1075-86.

Kong M,Hong SE (2014). Clinical outcome of helical tomotherapy for inoperable non-small cell lung cancer: the kyung hee university medical centre experience. Asian Pacific J Cancer Prev, 15, 1545-49.

Lawrence TS, Hayman J, Martel M, et al (1998). Regarding predicting radiation response. Int J Radiat Oncol Biol Phys, 41, 972-3.

Liu YC, Zhou SB, Gao F, et al (2013). Chemotherapy and late course three dimensional conformal radiotherapy for treatment of patients with stage III non-small cell lung cancer. Asian Pac J Cancer Prev, 14, 2663-65.

Malik Ps, Malik A, Deo SV, et al (2014). Underutilization of curative treatment among patients with non small cell lung cancer :experience from a tertiary care centre in india. Asian Pac J Cancer Prev, 15, 2875-78.

Mehta V (2005). Radiation pneumonitis and pulmonary fibrosis in non-small-cell lung cancer: Pulmonary function, prediction and prevention. Int J Radiation Oncology Biol Phys, 63, 5-24.

Ramella S, Trodella L, Mineo TC (2010). Adding ipsilateral V20 and V30 to conventional dosimetric constraints predicts radiation pneumonitis in stage IIIA-B NSCLC treated with combined-modality therapy. Int J Radiat Oncol Biol Phys, 76, 110-5.

Rancati T, Ceresolib GL, Gagliardic G (2003). Factors predicting radiation pneumonitis in lung cancer patients: a retrospective study. Radiotherapy and Oncology, 67, 275-83.

Rengan R, Chetty I, Decker R, et al (2013). Lung Cancer. In:Halperin EC, Wazer DE, Perez CA et al., Editors. Perez and Brady's Principles and Practice of Radiation Oncology.6th ed.Philadelphia: Lippincott Williams and Wilkins, 938-73.

Schallenkamp JM, Miller RC, Brinkmann DH, et al (2007). Incidence of radiation pneumonitis after thoracic irradiation: Dose-volume correlates. Int J Radiat Oncol Biol Phys, 67, 410-6.

Tsujino K, Hirota S, Endo M, et al (2003). Predictive value of dose-volume histogram parameters for predicting radiation pneumonitis after concurrent chemoradiation for lung cancer. Int J Radiat Oncol Biol Phys, 55, 110-5.

Wang S, Liao Z, Wei X, et al (2006). Analysis of clinical and dosimetric factors associated with treatment-related pneumonitis (TRP) in patients with non-small-cell lung cancer (NSCLC) treated with concurrent chemotherapy and three-dimensional conformal radiotherapy (3D-CRT). Int $J$ Radiat Oncol Biol Phys, 66, 1399-407.

Wang Z,Qiao YL,Tian X, et al (2012). Toll-like receptor 5 agonismprotects mice from radiation pneumonitis and pulmonary fibrosis. Asian Pac J Cancer Prev, 13, 4763-67. 